\title{
Bohmian picture of Rydberg atoms
}

\author{
Partha Ghose and Manoj K. Samal \\ S. N. Bose National Centre for Basic Sciences, Block JD, Sector III, Salt Lake, Kolkata 700098 \\ Animesh Datta \\ Department of Electrical Engineering, Indian Institute of Technology, Kanpur, 208016
}

\begin{abstract}
Unlike the previous theoretical results based on standard quantum mechanics that has established the nearly elliptical shapes for the centre-of-mass motion using numerical simulations, we show analytically that the Bohmian trajectories in Rydberg atoms are nearly elliptical.
\end{abstract}

\section{INTRODUCTION}

Ever since the advent of quantum mechanics one of the fundamental problems in physics has been to elucidate the transition from quantum to classical mechanics. After Schrödinger introduced coherent states in quantum mechanics [1], it became clear that they are optimal quantum mechanical states to describe the classical limit. Recently, there has been a renewed interest in this problem due to the experimental study of Rydberg atoms in external fields which provide a tool to study the quantum-classical transition regime [2,3]. These experiments [8] have shown that high quantum number states have the property of showing classical behaviour. Also, higher $n$ values lead to an evershrinking energy gap $E_{n}=-1 / 2 n^{2}$, and the energy spectrum approaches the continuum. Moreover, it is evident [4] that the highest angular momentum state $l=n-1=l_{n}$ in an atom has the special property of being a minimum uncertainty state.

In this paper, we study the problem from the point of view of the de Broglie-Bohm theory [7] to see the relationship between Bohmian trajectories and Kepler orbits in Rydberg atoms.

\section{STANDARD QUANTUM MECHANICAL ANALYSIS}

It has been shown that wave-packet solutions of the Schrödinger equation for the Coulomb problem travel along classical elliptic orbits of fixed mean eccentricity and angular momentum [4. These wave-packets are coherent states that have minimal quantum fluctuations in the non-commuting components of the Runge-Lenz vector in the plane of the orbit [5]. The study of the Runge-Lenz vector in quantum mechanics was first undertaken by Pauli [6] who used it to solve the Hydrogen atom problem.

In the asymptotic regime of large quantum numbers $n \approx l_{0}$, the WKB approximation of the radial wave-function of the hydrogen atom in 3 -dimensions is given by

$$
\begin{aligned}
\psi^{\delta}(r, \theta, \phi, t) & \approx\left(\frac{2 \omega_{0}}{\pi p_{0}(r)}\right)^{1 / 2} \exp \left[i S_{0}(r)\right]\left(\frac{\delta l_{0}}{\pi}\right)^{1 / 4} \exp \left[-(\theta-\pi / 2)^{2} \frac{\delta l_{0}}{2}\right] \\
& \times \sum_{\mu=-\infty}^{+\infty} \exp \left[i \delta l_{0}(\phi+2 \pi \mu)-\left(\phi+2 \pi \mu-\phi_{0}(r)\right)^{2} \frac{l_{0}}{2}\left(1-\delta^{2}\right)\right] \\
& \times \frac{1}{\left(2 \pi \alpha_{0}(r, t)\right)^{1 / 2}} \exp \left[\frac{-\left(\delta\left(\phi+2 \pi \mu-\phi_{0}(r)\right)-\frac{t-t_{0}(r)}{l_{0}^{3}}\right)^{2}}{2 \alpha_{0}(r, t)}\right]
\end{aligned}
$$

where the classical action in radial coordinates is given by

$$
S_{0}(r)=\int^{r} p_{0}\left(r^{\prime}\right) d r^{\prime}
$$

with the mean radial momentum

$$
p_{0}(r)=\left(2 E_{n_{0}}-\frac{l_{0}^{2}}{r^{2}}+\frac{2}{r}\right)^{1 / 2}
$$

In classical mechanics the quantities $\phi_{0}(r)$ and $t_{0}(r)$ are related to the action $S_{0}$ by 


$$
\phi_{0}(r)=-\frac{\partial S_{0}}{\partial l_{0}} \text { and } t_{0}(r)=\frac{\partial S_{0}}{\partial E_{n_{0}}} .
$$

The rate of spreading of the wave-packet is given by the complex width

$$
\alpha_{0}(r, t, \delta)=\frac{1}{2 \sigma^{2}}-i\left(\frac{3 t}{l_{0}^{4}}+2 f_{0}(r)\right)
$$

where $f_{0}(r)=\partial^{2} S / \partial E_{n}^{2}$. It follows from eqn. (1) that the quantum mechanical phase is

$$
S=S_{0}+\delta l_{0} \phi+\frac{1}{2} \arctan (b / a)+\frac{\lambda^{2}}{4 \gamma}-\frac{A^{2} b}{2\left(a^{2}+b^{2}\right)}
$$

where

$$
A=\delta\left(\phi-\phi_{0}(r)\right)-\frac{1}{l_{0}^{3}}\left(t-t_{0}(r)\right), \quad \gamma=\frac{2 \pi^{2} b \delta^{2}}{\left(a^{2}+b^{2}\right)}, \quad \lambda=\frac{\pi \delta A}{\left(a^{2}+b^{2}\right)}
$$

with

$$
a=\frac{1}{2 \sigma^{2}} \text { and } \quad b=\frac{3 t}{l_{0}^{4}}+2 f_{0}(r) .
$$

\section{BOHMIAN MECHANICAL ANALYSIS}

Let us now examine the problem of coherent states from the perspective of the de-Broglie-Bohm theory which is quite close to the classical Hamilton-Jacobi theory. In this theory the quantum mechanical action $S$ given by (6) satisfies the modified Hamilton-Jacobi equation

$$
\frac{\partial S}{\partial t}+\frac{(\nabla S)^{2}}{2 m}+Q+V=0
$$

where

$$
Q=-\frac{\hbar^{2}}{2 m} \frac{\nabla^{2} R}{R}
$$

and the wavefunction, written in the polar form $\psi=R e^{i S / \hbar}$, satisfies the Scrödinger equation

$$
i \hbar \frac{\partial \psi}{\partial t}=\left[-\frac{\hbar^{2}}{2 m} \nabla^{2}+V(x)\right] \psi
$$

In Bohmian mechanics one introduces the position as an additional variable (the so-called "hidden variable") through the guidance conditions [9]

$$
v_{r}=\frac{\partial S}{\partial r}, v_{\theta}=\frac{1}{r} \frac{\partial S}{\partial \theta}, v_{\phi}=\frac{1}{r \sin \theta} \frac{\partial S}{\partial \phi}
$$

Using these conditions, we can readily obtain the Bohmian trajectories of electrons in a Rydberg atom with high $l$ wave packets provided we also use the result that the radius of the $n$th Bohr orbit in a hydrogen atom is given by $r_{n} \approx n^{2}$. This is the expectation of the radius or its space averaged value. The time averaged value is the same. Using the expression for $S$ given by (6), we finally obtain

$$
\begin{aligned}
& \frac{\partial S}{\partial r}=p_{0}+\mathcal{O}\left(l_{0}^{-7}\right)+\mathcal{O}\left(l_{0}^{-14}\right) \\
& \frac{\partial S}{\partial \theta}=0 \\
& \frac{\partial S}{\partial \phi}=\delta l_{0}+\mathcal{O}\left(l_{0}^{-5}\right)
\end{aligned}
$$


The corresponding expressions in classical mechanics are

$$
\begin{aligned}
& \frac{\partial S}{\partial r}=p_{0} \\
& \frac{\partial S}{\partial \phi}=\delta l_{0}
\end{aligned}
$$

and these are known to lead to the equation of an ellipse 10]. Eqns. (13) and (14) show that the Bohmian trajectories of Rydberg atoms with $l_{0} \approx 50-100$ are ellipses for all practical purposes.

\section{CONCLUDING REMARKS}

The advantage of the Bohmian analysis we have carried out is that one can see clearly and analytically that the Bohmian trajectories in Rydberg atoms are nearly elliptical. Previous theoretical results using standard quantum mechanics have only been able to establish the nearly elliptical shapes for the centre-of-mass motion using numerical simulations [1].

\section{ACKNOWLEDGEMENTS}

PG and MKS acknowledge financial support from the Department of Science \& Technology, Govt. of India for this work. AD is grateful to the S. N. Bose National Centre for Basic Sciences for providing facilities for a summer project that enabled this work to be undertaken.

[1] E. Schrödinger, Naturwissenchafter 14, 137 (1927).

[2] R. F. Stebbings and F. B. Dunning, Rydberg States of Atoms and Molecules, Cambridge University Press, London, 1983.

[3] G. Alber and P. Zoller, Laser Excitation of Electronic Wave Packets in Rydberg Atoms Physics Report, 199(5), 1(1991).

[4] M. Nauenberg, Phys. Rev. A 40(2), 1133 (1989).

[5] C. Runge, Vektoranalysis, Vol. I, p.70, S. Hirzel, Leipzig, 1919; W. Lenz, Z. Physik, 24, 197(1924).

[6] W. Pauli, Z. Phys., 36, 336 (1926).

[7] D. Bohm and B. J. Hiley, The Undivided Universe, Routledge and Chapman and Hall, 1993.

[8] J. A. Yeazell and C. R. Stroud, Phys. Rev. Lett., 60, 494 (1988).

[9] P. R. Holland, Quantum Theory of Motion, Cambridge University Press, 1993.

[10] H. Goldstein, Classical Mechanics, Addison-Wesley, 1968.

[11] P. Bellomo and C. R. Stroud Jr., Classical evolution of Quantum elliptic states, arXiv:quant-ph/9812022.

[12] P. Ghose and Manoj K. Samal, Smooth transition between classical and quantum mechanics, arXiv:quant-ph/0104105. 